Алгебра и анализ

Toм 20 (2008), № 4
St. Petersburg Math. J.

Vol. 20 (2009), No. 4, Pages 553-567

S 1061-0022(09)01061-9

Article electronically published on June 1, 2009

\title{
SOME REMARKS ON SPHERICAL HARMONICS
}

\author{
V. M. GICHEV
}

\begin{abstract}
Several observations on spherical harmonics and their nodal sets are presented: a construction for harmonics with prescribed zeros; a natural representation for harmonics on $\mathbb{S}^{2}$; upper and lower bounds for the nodal length and the inner radius (the upper bounds are sharp); the sharp upper bound for the number of common zeros of two spherical harmonics on $\mathbb{S}^{2}$; the mean Hausdorff measure of the intersection of $k$ nodal sets for harmonics of different degrees on $\mathbb{S}^{m}$, where $k \leq m$ (in particular, the mean number of common zeros of $m$ harmonics).
\end{abstract}

\section{INTRODUCTION}

This article contains several observations on spherical harmonics and their nodal sets; the emphasis is on the case of $\mathbb{S}^{2}$.

Let $M$ be a compact connected homogeneous Riemannian manifold, let $G$ be a compact Lie group acting on $M$ transitively by isometries, and let $\mathcal{E}$ be a $G$-invariant subspace of the (real) eigenspace for some nonzero eigenvalue of the Laplace-Beltrami operator. We show that each function in $\mathcal{E}$ can be realized as the determinant of a matrix whose entries are values of the reproducing kernel for $\mathcal{E}$. There is a similar well-known construction for orthogonal polynomials. However, the method does not work in the case of an arbitrary finite-dimensional $G$-invariant subspace of $C(M)$ (see Remark 2). There is a natural and unique (up to scaling factors) realization of this type for spherical harmonics on $\mathbb{S}^{2}$. It can be obtained by complexification and restriction to the null-cone $x^{2}+y^{2}+z^{2}=0$ in $\mathbb{C}^{3}$. There is a two-sheeted equivariant covering of this cone by $\mathbb{C}^{2}$, which identifies the space $\mathcal{H}_{n}$ of harmonic homogeneous complex-valued polynomials of degree $n$ on $\mathbb{R}^{3}$ with the space $\mathcal{P}_{2 n}^{2}$ of homogeneous holomorphic polynomials on $\mathbb{C}^{2}$ of degree $2 n \mathbb{1}$

The set of all zeros of a real spherical harmonic $u$ is called a nodal set. We say that $u$ and its nodal set $N_{u}$ are regular if 0 is not a critical value of $u$. Then each component of $N_{u}$ is a Jordan contour. In accordance with [1], any two nodal sets $N_{u}, N_{v}$, where $u, v \in \mathcal{H}_{n}^{\mathbb{R}}$ and $n>0$, have a nonempty intersection; moreover, if $u$ is regular, then each component of $N_{u}$ contains at least two points of $N_{v}$. The set $N_{u} \cap N_{v}$ may be infinite, but the family of such pairs $(u, v)$ is closed and nowhere dense in $\mathcal{H}_{n}^{\mathbb{R}} \times \mathcal{H}_{n}^{\mathbb{R}}$. If $N_{u} \cap N_{v}$ is finite, then card $N_{u} \cap N_{v} \leq 2 n^{2}$. This estimate follows from the Bezout theorem and is sharp. This gives an upper bound (probably, not sharp) for the number of critical points of a generic spherical harmonic. The configuration of critical points is always

2000 Mathematics Subject Classification. Primary 33E30.

Key words and phrases. Nodal set, spherical harmonics, Hausdorff measure.

Supported in part by RFBR (grant nos. 06008-01403 and 06007-8951), and also by Sibirean Department of RAS (project no. 117).

${ }^{1}$ In 1876, Sylvester used an equivalent construction to refine Maxwell's method for representation of spherical harmonics. In accordance with it, we must differentiate the function $1 / r$, where $r$ is the distance to the origin, in suitable directions in $\mathbb{R}^{3}$ to get a real harmonic. The directions are determined uniquely; the corresponding points in $\mathbb{S}^{2}$ are called the poles (see [15, Chapter 9] or 31 11.5.2]; the books [7. Chapter 7, § 5] and [1. Appendix A] contain extended expositions and further information). 
degenerate in a sense (see Remark 5). The problem of finding lower bounds seems to be more difficult. Partial results and computer experiments show that $2 n$ may be the sharp lower bound.

The investigation of metric and topological properties of nodal sets has a long and rich history; we only give a few remarks on the subject of this paper. Let $\Delta$ be the Laplace-Beltrami operator, and let $\lambda$ be an eigenvalue of $-\Delta$.

In 1978, Brüning 5] found a lower bound of the form $c \sqrt{\lambda}$ for the length of a nodal set on a Riemann surface. Yau conjectured (see [22, Problem 74]) that the Hausdorff measure of the nodal set of a $\lambda$-eigenfunction on a compact Riemannian manifold admits upper and lower bounds of type $c \sqrt{\lambda}$. This conjecture was proved by Donnelly and Fefferman in [8] for real analytic manifolds. In [18], Savo proved that $\frac{1}{11} \operatorname{Area}(M) \sqrt{\lambda}$ is a lower bound for the length of a nodal set in a surface $M$ for all sufficiently large $\lambda$ (and for all $\lambda$ if the curvature is nonnegative). Upper and lower estimates of the inner radius were found by Mangoubi (see [13, 14]); in the case of surfaces, they are of order of $\lambda^{-\frac{1}{2}}$ (see [13]).

The 1-dimensional Hausdorff measure of a set on $\mathbb{S}^{2}$ can be found by integrating over $\mathrm{SO}(3)$ the counting function for the number of its common points with the translates of a suitable subset of $\mathbb{S}^{2}$ (see Theorem 4). Using estimates for the number of common zeros, we give upper and lower bounds for the length of a nodal set and for the inner radius of a nodal domain in $\mathbb{S}^{2}$. The upper bounds are sharp.

Let $\mathcal{H}_{n}^{m+1}$ be the space of all real spherical harmonics of degree $n$ on the unit sphere $\mathbb{S}^{m}$ in $\mathbb{R}^{m+1}$. Assigning to a point of $\mathbb{S}^{m}$ the evaluation functional at it on $\mathcal{H}_{n}^{m+1}$, we get an equivariant immersion of $\mathbb{S}^{m}$ to the unit sphere in $\mathcal{H}_{n}^{m+1}$, which is locally a metric homothety with the coefficient $\sqrt{\frac{\lambda_{n}}{m}}$, where $\lambda_{n}=n(n+m-1)$ is the eigenvalue of $-\Delta$ in $\mathcal{H}_{n}^{m+1}$. This makes it possible to calculate the mean Hausdorff measure of the intersection of the nodal sets of $k$ harmonics of degrees $n_{1}, \ldots, n_{k}$ : it is equal to $c \sqrt{\lambda_{n_{1}} \cdots \lambda_{n_{k}}}$, where $c$ depends only on $m$ and $k$ and $k \leq m$ (Theorem 6). In particular, if $k=m$, then we get the mean number of common zeros of $m$ harmonics, equal to $2 m^{-\frac{m}{2}} \sqrt{\lambda_{n_{1}} \cdots \lambda_{n_{m}}}$; if $m=2$, then we get $\sqrt{\lambda_{n_{1}} \lambda_{n_{2}}}$. In [8], Donnelly and Fefferman wrote: "A main theme of this paper is that a solution of $\Delta F=-\lambda F$, on a real analytic manifold, behaves like a polynomial of degree $c \sqrt{\lambda}$." Following this idea, L. Polterovich conjectured that the mean number of common zeros is subject to the Bézout theorem, i.e., that it is as above. Thus, the result for $k=m$ confirms this conjecture up to multiplication by a constant, and may be treated as "the Bézout theorem in the mean" for the spherical harmonics. For $k=1$, by different but similar methods, the mean Hausdorff measure was found by Bérard in [4] and Neuheisel in [16]. The case of a flat torus was investigated by Rudnick and Wigman in [17].

\section{§1. Construction of EIGENFunCtions that VANish ON PRESCRIBED Finite SETS}

In this section, $M$ is a compact connected oriented homogeneous Riemannian manifold of a compact Lie group $G$ acting by isometries on $M, \Delta$ is the Laplace-Beltrami operator on $M$,

$$
\lambda>0
$$

is an eigenvalue of $-\Delta, \mathcal{E}_{\lambda}$ is the corresponding real eigenspace (i.e., $\mathcal{E}_{\lambda}$ consists of realvalued eigenfunctions), and $\mathcal{E}$ is its $G$-invariant linear subspace. Thus, $\mathcal{E}$ is a finite sum of $G$-invariant irreducible subspaces of $C^{\infty}(M)$. The invariant measure with the total mass 1 on $M$ is denoted by $\sigma, L^{2}(M)=L^{2}(M, \sigma)$. For any $a \in M$, there exists a unique 
$\phi_{a} \in \mathcal{E}$ that realizes the evaluation functional at $a:\left\langle u, \phi_{a}\right\rangle=u(a)$ for all $u \in \mathcal{E}$. Put

$$
\phi(a, b)=\phi_{a}(b), \quad a, b \in M \text {. }
$$

It follows that

$$
\begin{aligned}
& \phi(a, b)=\phi_{a}(b)=\left\langle\phi_{a}, \phi_{b}\right\rangle=\left\langle\phi_{b}, \phi_{a}\right\rangle=\phi_{b}(a)=\phi(b, a), \\
& u(x)=\left\langle u, \phi_{x}\right\rangle=\int \phi(x, y) u(y) d \sigma(y) \text { for all } u \in \mathcal{E}, \\
& x \in N_{u} \Longleftrightarrow \phi_{x} \perp u, \\
& \phi_{x} \neq 0 \text { for all } x \in M .
\end{aligned}
$$

The last relation holds true by the homogeneity of $M$. By (3), $\phi(x, y)$ is the reproducing kernel for $\mathcal{E}$ (i.e., the mapping $u(x) \rightarrow \int \phi(x, y) u(y) d \sigma(y)$ is the orthogonal projection onto $\mathcal{E}$ in $\left.L^{2}(M)\right)$.

Let $a_{1}, \ldots, a_{k}, x, y \in M$. Let $a=\left(a_{1}, \ldots, a_{k}\right) \in M^{k}$, and let $a$ also denote the corresponding $k$-subset of $M: a=\left\{a_{1}, \ldots, a_{k}\right\}$. Set

$$
\Phi_{k}^{a}(x, y)=\Phi_{k, y}^{a}(x)=\operatorname{det}\left(\begin{array}{cccc}
\phi\left(a_{1}, a_{1}\right) & \ldots & \phi\left(a_{1}, a_{k}\right) & \phi\left(a_{1}, y\right) \\
\vdots & \ddots & \vdots & \vdots \\
\phi\left(a_{k}, a_{1}\right) & \ldots & \phi\left(a_{k}, a_{k}\right) & \phi\left(a_{k}, y\right) \\
\phi\left(x, a_{1}\right) & \ldots & \phi\left(x, a_{k}\right) & \phi(x, y)
\end{array}\right) .
$$

Obviously, $\Phi_{k}^{a}(x, y)=\Phi_{k}^{a}(y, x)$. We fix $y$ and set $v=\Phi_{k, y}^{a}$. Then, by (6),$v \in \mathcal{E}$ and

$$
a_{1}, \ldots, a_{k} \in N_{v} \text {. }
$$

We say that $a_{1}, \ldots, a_{k}$ are independent if the vectors $\phi_{a_{1}}, \ldots, \phi_{a_{k}} \in \mathcal{E}$ are linearly independent. For a subset $X \subseteq M$, put

$$
\mathcal{N}_{X}=\operatorname{span}\left\{\phi_{x}: x \in X\right\} .
$$

If $X=N_{u}$, where $u \in \mathcal{E}$, then we abbreviate: $\mathcal{N}_{N_{u}}=\mathcal{N}_{u}$. Set $n=\operatorname{dim} \mathcal{E}-1$. Condition (11) implies that $n \geq 1$ (note that $\mathcal{E}$ is real and $G$-invariant).

Lemma 1. Let $a \in M^{k}$, where $k \leq n$. Then $a_{1}, \ldots, a_{k}$ are independent if and only if $\Phi_{k, y}^{a} \neq 0$ for some $y \in M$.

Proof. By (44), we have $\mathcal{E}=\mathcal{N}_{M}$, and $\mathcal{N}_{a} \neq \mathcal{E}$ because $k \leq n$. Therefore, if $a_{1}, \ldots, a_{k}$ are independent, then we get an independent set by adding $y$ to $a$, for some $y \in M$. Then $\Phi_{k, y}^{a} \neq 0$ because $\Phi_{k, y}^{a}(y)>0$ (by (2) and (6), $\Phi_{k, y}^{a}(y)$ is the determinant of the Gram matrix for the vectors $\left.\phi_{a_{1}}, \ldots, \phi_{a_{k}}, \phi_{y}\right)$. Clearly, $\Phi_{k, y}^{a}=0$ for all $y \in M$ if $a_{1}, \ldots, a_{k}$ are dependent.

The following proposition implies that each function in $\mathcal{E}$ can be realized in the form (6).

Proposition 1. For any $u \in \mathcal{E}, \mathcal{N}_{u}=u^{\perp} \cap \mathcal{E}$.

Lemma 2. If $u, v \in \mathcal{E}$ and $N_{v} \supseteq N_{u}$, then $v=c u$ for some $c \in \mathbb{R}$.

Proof. This immediately follows from the inclusion $N_{v} \supseteq N_{u}$ and Lemma 1 in [1], which states that $v=c u$ for some $c \in \mathbb{R}$ if there exist nodal domains $U, V$ for $u, v$, respectively, such that $V \subseteq U$.

Here is a sketch of the proof of this lemma; it is based on the same idea as Courant's nodal domain theorem. Since $u$ does not change its $\operatorname{sign}$ in $U, \lambda$ is the first Dirichlet eigenvalue for $U$. Hence, it has multiplicity 1 and $D(w) \geq \lambda\|w\|_{L^{2}(U)}$ for all $w \in C^{2}(M)$ that vanish on $\partial U$, where $D$ is the Dirichlet form on $U$. Moreover, equality occurs if and 
only if $w=c u$ for some $c \in \mathbb{R}$. On the other hand, if $w$ vanishes outside $V$ and coincides with $v$ in $V$, then we have equality.

Proof of Proposition 1. If $v \in \mathcal{E}$ and $v \perp \mathcal{N}_{u}$, then $N_{v} \supseteq N_{u}$ by (4). Thus, $v \in \mathbb{R} u$ by Lemma 2, Therefore, $\mathcal{N}_{u} \supseteq u^{\perp} \cap \mathcal{E}$. The reverse inclusion is evident.

Let $\Phi: M^{n+1} \rightarrow \mathcal{E}$ be the mapping $(a, y) \mapsto \Phi_{n, y}^{a}$; we set $\mathcal{U}=\Phi\left(M^{n+1}\right)$.

Theorem 1. (i) Let $u \in \mathcal{E}, u \neq 0$. For $(a, y) \in N_{u}^{n} \times M$, we have

$$
\Phi(a, y)=c(a, y) u
$$

where $c$ is a continuous nontrivial function on $N_{u}^{n} \times M$.

(ii) $\mathcal{U}$ is a compact symmetric neighborhood of zero in $\mathcal{E}$.

(iii) For every $a \in M^{n}$, there exists a nontrivial nodal set containing a; for a generic point $a$, this set is unique.

Proof. Let $a \in N_{u}^{n}$. If $a_{1}, \ldots, a_{n}$ are independent, then $\operatorname{codim} \mathcal{N}_{a}=1$; since $u \perp \mathcal{N}_{u}$ by (4), we get (9), where $c(a, y) \neq 0$ for some $y \in M$ by Lemma 1. If $a_{1}, \ldots, a_{n}$ are dependent, then $\Phi(a, y)=0$ for all $y \in M$ by the same lemma. The function $c$ is continuous by (6); it is nonzero because the set $N_{u}$ contains independent points $a_{1}, \ldots, a_{n}$ by Proposition 1. This proves (i).

By (6), $\Phi$ is continuous. Hence, $\mathcal{U}$ is compact. Since $M$ is connected, for any $u \in \mathcal{U}$ we may get the segment $[0, u]$ by moving $y$; hence, $\mathcal{U}$ is starlike. Since transposition of every two points in $a$ changes the sign of $c(a, y), \mathcal{U}$ is symmetric if $n>1$; for $n=1$, $\mathcal{U}$ is a disk in $\mathcal{E}$ because it is $G$-invariant and starlike. Thus, $\mathcal{U}$ is compact, symmetric, starlike, and $\bigcup_{t>0} t \mathcal{U}=\mathcal{E}$. Hence, $\mathcal{U}$ is a neighborhood of zero, i.e., (ii) is true.

For $a \in M^{n}$, let $a^{\prime} \subseteq a$ be a maximal independent subset of $a$. Then $\Phi_{k, y}^{a^{\prime}} \neq 0$ for some $y \in M$ by Lemma 1, where $k=\operatorname{card} a^{\prime}$. Set $v=\Phi_{k, y}^{a^{\prime}}$. By (7), we have $a^{\prime} \subset N_{v}$. By (4), $N_{v}$ contains any point $x \in M$ such that $\phi_{x} \in \mathcal{N}_{a^{\prime}}$. Hence, $N_{v}$ includes $a$. The set $N_{v}$ is unique if $a_{1}, \ldots, a_{n}$ are independent, because $\operatorname{codim} \mathcal{N}_{v}=1$ in this case. Since $M$ is homogeneous and $\mathcal{E}$ is finite-dimensional, the functions $\phi_{x}, x \in M$, are real analytic. Therefore, either $\Phi_{n, y}^{a}=0$ for all $(a, y) \in M^{n+1}$, or $\Phi_{n, y}^{a} \neq 0$ for generic $(a, y)$ (note that $M$ is connected). Finally, $\Phi_{n, y}^{a} \neq 0$ for some $(a, y) \in M^{n+1}$, because $\mathcal{N}_{M}=\mathcal{E}$ due to (4) and (5).

A closed subset $X \subseteq M$ is called an interpolation set for a function space $\mathcal{F} \subseteq C(M)$ if $\left.\mathcal{F}\right|_{X}=C(X)$.

Corollary 1. Let $k \leq \operatorname{dim} \mathcal{E}$. For generic $a_{1}, \ldots, a_{k} \in M$, the set $a=\left\{a_{1}, \ldots, a_{k}\right\}$ is an interpolation set for $\mathcal{E}$.

Remark 1. The function $c$ may vanish on some components of the set $N_{u}^{n} \times M$. For example, let $M$ be the unit sphere $\mathbb{S}^{2} \subset \mathbb{R}^{3}$, and let $\mathcal{E}$ be the restriction to it of the space of harmonic homogeneous polynomials of degree $k$; then $\operatorname{dim} \mathcal{E}=2 k+1, n=2 k$. If $k>1$, then any big circle $\mathbb{S}^{1}$ in $\mathbb{S}^{2}$ is contained in several nodal sets (for example, the nodal sets of the functions $x_{1} f\left(x_{2}, x_{3}\right)$, where $f$ is harmonic, contain the big circle $\left.\left\{x_{1}=0\right\} \cap \mathbb{S}^{2}\right)$; moreover, if $k$ is odd, then $\mathbb{S}^{1}$ may be a component of $N_{u}$. Hence, $\operatorname{codim} \mathcal{N}_{\mathbb{S}^{1}}>1$ and $\Phi(a, y)=0$ for all $(a, y) \in\left(\mathbb{S}^{1}\right)^{n} \times \mathbb{S}^{2}$.

Remark 2. Theorem 1 fails for a generic finite-dimensional $G$-invariant subspace $\mathcal{E} \subseteq$ $C(M)$. Indeed, if $\operatorname{dim} \mathcal{E}>1$ and $\mathcal{E}$ contains constant functions, then it includes the open subset of functions without zeros, which evidently cannot be realized in the form (6). Furthermore, the theorem implies that the products $\phi_{a_{1}} \wedge \cdots \wedge \phi_{a_{n}}$ fill a neighborhood of zero in the $n$th exterior power of $\mathcal{E}$, which can be identified with $\mathcal{E}$. Obviously, this 
property implies the interpolation property of Corollary 1, but the converse is not true; an example is the space of all homogeneous polynomials of degree $m>1$ on $\mathbb{R}^{3}$, restricted to $\mathbb{S}^{2}$ (or the space of all polynomials of degree less than $n$ on $[0,1] \subset \mathbb{R}$, where $n>2$ ).

\section{§. Spherical Harmonics ON $\mathbb{S}^{2}$}

Let $\mathcal{P}_{n}^{m}$ denote the space of all homogeneous holomorphic polynomials of degree $n$ on $\mathbb{C}^{m}$ and/or the space of all complex-valued homogeneous polynomials of degree $n$ on $\mathbb{R}^{m}$; clearly, there is a one-to-one correspondence between these spaces. Its subspace of polynomials that are harmonic on $\mathbb{R}^{m}$ is denoted by $\mathcal{H}_{n}^{m}$; we omit the index $m$ in $\mathcal{H}_{n}^{m}$ if $m=3$. Then $\operatorname{dim} \mathcal{H}_{n}=2 n+1$. The polynomials in $\mathcal{H}_{n}^{m}$, as well as their traces on the unit sphere $\mathbb{S}^{m-1} \subset \mathbb{R}^{m}$, are called spherical harmonics. They are eigenfunctions of the Laplace-Beltrami operator; if $m=3$, then the corresponding eigenvalue is $-n(n+1)$. For the proof of these facts, see, e.g., [19. We say that $u \in \mathcal{P}_{n}^{m}$ is real if it takes real values on $\mathbb{R}^{m}$.

The standard inner product in $\mathbb{R}^{m}$ and its bilinear extension to $\mathbb{C}^{m}$ will be denoted by $\langle$,$\rangle , and$

$$
r(v)=|v|=\sqrt{\langle v, v\rangle}, \quad v \in \mathbb{R}^{m},
$$

so that $r^{2}$ is a holomorphic quadratic form on $\mathbb{C}^{m}$. For $a \in \mathbb{C}^{m}$, set

$$
l_{a}(v)=\langle a, v\rangle .
$$

The functions $\Phi_{k}^{a}(x, y)$ admit holomorphic extensions relative to all variables (except for $k$ ). If $M=\mathbb{S}^{2} \subset \mathbb{R}^{3}$, then the extension to $\mathbb{C}^{3}$ and subsequent restriction to the null-cone

$$
S_{0}=\left\{z \in \mathbb{C}^{3}: r^{2}(z)=0\right\}
$$

makes it possible to construct a kind of a natural representation in the form (6), which is unique up to multiplication by a complex number, for any complex-valued spherical harmonic. The projection of $S_{0}$ to $\mathbb{C P}^{2}$ is the Riemann sphere $\mathbb{C P}^{1}$. The cone $S_{0}$ admits a natural parametrization:

$$
\kappa\left(\zeta_{1}, \zeta_{2}\right)=\left(z_{1}, z_{2}, z_{3}\right)=\left(2 \zeta_{1} \zeta_{2}, \zeta_{1}^{2}-\zeta_{2}^{2}, i\left(\zeta_{1}^{2}+\zeta_{2}^{2}\right)\right), \quad \zeta_{1}, \zeta_{2} \in \mathbb{C} .
$$

Lemma 3. The mapping $R: \mathcal{H}_{n} \rightarrow \mathcal{P}_{2 n}^{2}$ defined by

$$
R p=p \circ \kappa
$$

is one-to-one and intertwines the natural representations of $\mathrm{SO}(3)$ and $\mathrm{SU}(2)$ in $\mathcal{H}_{n}$ and $\mathcal{P}_{2 n}^{2}$, respectively.

Proof. Clearly, $p \circ \kappa$ is a homogeneous polynomial on $\mathbb{C}^{2}$ of degree $2 n$ for any $p \in \mathcal{P}_{n}^{3}$. Further, $\kappa$ is equivariant with respect to the natural actions of $\mathrm{SU}(2)$ in $\mathbb{C}^{2}$ and $\mathrm{SO}(3)$ in $\mathbb{C}^{3}$ : an easy calculation with (10) shows that the change of variables $\zeta_{1} \rightarrow a \zeta_{1}+b \zeta_{2}$, $\zeta_{2} \rightarrow-\bar{b} \zeta_{1}+\bar{a} \zeta_{2}$, where $|a|^{2}+|b|^{2}=1$, induces a linear transformation in $\mathbb{C}^{3}$ that preserves $r^{2}$ and leaves $\mathbb{R}^{3}$ invariant (in other words, the transformation of $\mathcal{P}_{2}^{2}$ induced by this change of variables in the base $2 \zeta_{1} \zeta_{2}, \zeta_{1}^{2}-\zeta_{2}^{2}, i\left(\zeta_{1}^{2}+\zeta_{2}^{2}\right)$ corresponds to a matrix in $\mathrm{SO}(3))$. Hence, $R$ is an intertwining operator. It is well known that

$$
\mathcal{P}_{n}^{3}=\mathcal{H}_{n} \oplus r^{2} \mathcal{P}_{n-2}^{3}
$$

(see, e.g., [19]). Since $R \neq 0$ and $R r^{2}=0$, we get $R \mathcal{H}_{n} \neq 0$. It remains to note that the natural representations of these groups in $\mathcal{H}_{n}$ and $\mathcal{P}_{n}^{2}$ are irreducible.

Corollary 2. For any $p \in \mathcal{H}_{n} \backslash\{0\}$, the set $p^{-1}(0) \cap S_{0}$ is a union of $2 n$ complex lines; some of them may coincide. If the lines are distinct, $q \in \mathcal{H}_{n}$, and $p^{-1}(0) \cap S_{0}=$ $q^{-1}(0) \cap S_{0}$, then $q=c p$ for some $c \in \mathbb{C}$. 
Proof. Clearly, $\kappa$ maps lines onto lines and induces an embedding of $\mathbb{C P}^{1}$ into $\mathbb{C P}^{2}$.

The functions $\phi_{a}$ of the previous section can be written explicitly:

$$
\phi_{a}(x)=c_{n} P_{n}(\langle a, x\rangle), \quad \text { where } a, x \in \mathbb{S}^{2},
$$

$c_{n}$ is a normalizing constant, and $P_{n}$ is the $n$th Legendre polynomial:

$$
P_{n}(t)=\frac{1}{2^{n} n !} \frac{d^{n}}{d t^{n}}\left(t^{2}-1\right)^{2 n}
$$

There is a unique extension of

$$
\phi(a, x)=\phi_{a}(x)
$$

to $\mathbb{R}^{3}$ that is homogeneous of degree $n$ and harmonic in both variables (it is also symmetric and extends to $\mathbb{C}^{3}$ holomorphically). For example, if $n=3$, then $2 P_{3}(t)=5 t^{3}-3 t$, and $\phi(a, x)$ is proportional to $5\langle a, x\rangle^{3}-3\langle a, a\rangle\langle a, x\rangle\langle x, x\rangle$ (if $a=(1,0,0)$, then this reduces to $2 x_{1}^{3}-3 x_{1} x_{2}^{2}-3 x_{1} x_{3}^{2}$ ). Of course, the representation of $p \in \mathcal{H}_{n}$ in the form (6) holds true for $M=\mathbb{S}^{2}$, but there is a more natural version in this case. For $\zeta=\left(\zeta_{1}, \zeta_{2}\right) \in \mathbb{C}^{2}$, set $j \zeta=\left(-\zeta_{2}, \zeta_{1}\right)$.

Theorem 2. Let $p \in \mathcal{H}_{n}$. Suppose that $p^{-1}(0) \cap S_{0}$ is the union of distinct lines $\mathbb{C} a_{1}, \ldots, \mathbb{C} a_{2 n}$. Then there exists a constant $c \neq 0$ such that

$$
p(x) p(y)=c \operatorname{det}\left(\begin{array}{cccc}
\left\langle a_{1}, a_{1}\right\rangle^{n} & \ldots & \left\langle a_{1}, a_{2 n}\right\rangle^{n} & \left\langle a_{1}, y\right\rangle^{n} \\
\vdots & \ddots & \vdots & \vdots \\
\left\langle a_{2 n}, a_{1}\right\rangle^{n} & \ldots & \left\langle a_{2 n}, a_{2 n}\right\rangle^{n} & \left\langle a_{2 n}, y\right\rangle^{n} \\
\left\langle x, a_{1}\right\rangle^{n} & \ldots & \left\langle x, a_{2 n}\right\rangle^{n} & \langle x, y\rangle^{n}
\end{array}\right)
$$

for all $y \in S_{0}, x \in \mathbb{C}^{3}$. Moreover, replacing $\langle x, y\rangle^{n}$ with $\phi(x, y)$ in the matrix, we get such a representation of $p(x) p(y)$ for all $x, y \in \mathbb{C}^{3}$ (with another $c$ in general).

Proof. A calculation shows that $\langle a, x\rangle^{n}$ is harmonic in $x$ for all $n$ if $a \in S_{0}$. Hence, the function $\Phi_{y}^{a}(x)=\Phi^{a}(x, y)$ on the right-hand side belongs to $\mathcal{H}_{n}$ for each $y \in S_{0}$. Clearly, $\Phi_{y}^{a}\left(a_{k}\right)=0$ for all $k=1, \ldots, 2 n$. By Corollary 2 , $\Phi_{y}^{a}$ is proportional to $p$. Since $\Phi^{a}(x, y)=\Phi^{a}(y, x)$, we get (11) if the right-hand side is nontrivial. Thus, we must prove that $c \neq 0$. Let $x \in S_{0}$. There exist $\alpha_{1}, \ldots, \alpha_{2 n}, \xi, \eta \in \mathbb{C}^{2}$ such that $a_{k}=\kappa\left(\alpha_{k}\right)$ for all $k$, $x=\kappa(\xi)$, and $y=\kappa(\eta)$. By a straightforward calculation, for any $a, b \in \mathbb{C}^{2}$ we get

$$
\langle\kappa(a), \kappa(b)\rangle=-2\langle a, j b\rangle^{2} .
$$

Hence, the right-hand side of (11) is equal to

$$
-2^{(2 n+1) n} c \operatorname{det}\left(\begin{array}{cccc}
\left\langle\alpha_{1}, j \alpha_{1}\right\rangle^{2 n} & \ldots & \left\langle\alpha_{1}, j \alpha_{2 n}\right\rangle^{2 n} & \left\langle\alpha_{1}, j \eta\right\rangle^{2 n} \\
\vdots & \ddots & \vdots & \vdots \\
\left\langle\alpha_{2 n}, j \alpha_{1}\right\rangle^{2 n} & \ldots & \left\langle\alpha_{2 n}, j \alpha_{2 n}\right\rangle^{2 n} & \left\langle\alpha_{2 n}, j \eta\right\rangle^{2 n} \\
\left\langle\xi, j \alpha_{1}\right\rangle^{2 n} & \ldots & \left\langle\xi, j \alpha_{2 n}\right\rangle^{2 n} & \langle\xi, j \eta\rangle^{2 n}
\end{array}\right) .
$$

The determinant can be calculated explicitly. More generally, if $C=\left(c_{r s}\right)_{r, s=1}^{k+1}$, where $c_{r s}=\left\langle a_{r}, b_{s}\right\rangle^{k}, a_{r}, b_{s} \in \mathbb{C}^{2}$, then

$$
\operatorname{det} C=\prod_{r=1}^{k}\left(\begin{array}{l}
k \\
r
\end{array}\right) \prod_{s<r}\left\langle a_{r}, j a_{s}\right\rangle \prod_{s<r}\left\langle b_{r}, j b_{s}\right\rangle .
$$

Let $a_{r}=\left(a_{r, 1}, a_{r, 2}\right), b_{s}=\left(b_{s, 1}, b_{s, 2}\right)$. If all the entries are nonzero, then

$$
c_{r s}=\sum_{t=0}^{k}\left(\begin{array}{l}
k \\
r
\end{array}\right)\left(a_{r, 1} b_{s, 1}\right)^{t}\left(a_{r, 2} b_{s, 2}\right)^{k-t}=a_{r, 2}^{k} b_{s, 1}^{k} \sum_{t=0}^{k}\left(\begin{array}{l}
k \\
r
\end{array}\right)\left(\frac{a_{r, 1}}{a_{r, 2}}\right)^{t}\left(\frac{b_{s, 2}}{b_{s, 1}}\right)^{k-t} \text {. }
$$


We can factor out common factors from rows and columns of $C$. Then we get a matrix $\tilde{C}$ that admits the decomposition $\tilde{C}=A B$, where

$$
A=\left(\left(\begin{array}{c}
k \\
r
\end{array}\right) \alpha_{r}^{t}\right)_{r, t=0}^{k}, \quad B=\left(\beta_{s}^{k-t}\right)_{t, s=0}^{k}, \quad \alpha_{r}=\frac{a_{r, 1}}{a_{r, 2}}, \quad \beta_{s}=\frac{b_{s, 2}}{b_{s, 1}} .
$$

Thus, the computation of $\operatorname{det} C$ is reduced to the Vandermonde determinant. A straightforward calculation proves (14); obviously, the assumption that the entries are nonzero is not essential. By (14) and (12), this implies that the determinant in (13) is nonzero if the lines $\mathbb{C} \xi, \mathbb{C} \eta, \mathbb{C} a_{1}, \ldots, \mathbb{C} a_{2 n}$ are distinct (if $a \in S_{0}$, then the plane $\langle z, a\rangle=0$ intersects $S_{0}$ along the line $\mathbb{C} a$ ). Hence, $c \neq 0$.

The definitions of $P_{n}$ and $\phi$ show that

$$
\phi(x, y)=s_{n}\langle x, y\rangle^{n}+r^{2}(x) r^{2}(y) h(x, y),
$$

where $s_{n}>0$ is a constant and $h$ is a polynomial. Therefore, we can get a function $f \neq 0$ on $\mathbb{C}^{3}$ that coincides with $p(x)$ on $S_{0}$ up to a constant factor, by replacing $\langle x, y\rangle^{n}$ with $\phi(x, y)$ in (11) and fixing a generic $y \in \mathbb{C}^{3}$. By Corollary 2, the same is true on $\mathbb{C}^{3}$ because $f \in \mathcal{H}_{n}$ by (11) (all functions in the last row are harmonic in $x$ ). Since $\phi(x, y)=\phi(y, x)$, this proves the second assertion.

Remark 3 . The set $p^{-1}(0) \cap S_{0}$, where $p \in \mathcal{H}_{n}$, is also distinguished by the orthogonality condition

$$
\int_{\mathbb{S}^{2}} p(x)\langle x, w\rangle^{n} d \sigma(x)=0,
$$

where $\sigma$ is the invariant measure on $\mathbb{S}^{2}$ and $w \in S_{0}$. This is a consequence of (15) because $\int p(x) \phi(x, y) d \sigma(x)=p(y)$ for all $y \in \mathbb{S}^{2}$, hence for all $y \in \mathbb{R}^{3}\left(p(y)\right.$ and $\phi_{x}(y)$ are homogeneous of degree $n$ ), and moreover, for all $y \in \mathbb{C}^{3}$ (both sides are holomorphic on $y$ ). In particular, this is true for $y \in S_{0}$, but $\phi(x, y)=s_{n}\langle x, y\rangle^{n}$ in this case.

If $p^{-1}(0) \cap S_{0}$ is the union of distinct lines $\mathbb{C} a_{k}, k=1, \ldots, 2 n$, then the functions $\left\langle x, a_{k}\right\rangle^{n}, k=1, \ldots, 2 n$, form a linear base for the space of functions in $\mathcal{H}_{n}$ that are orthogonal to $p$ with respect to the bilinear form $\int f g d \sigma$. This is a consequence of (12): it is easy to check that the functions $\left\langle\zeta, b_{s}\right\rangle^{k}$ on $\mathbb{C}^{2}$, where $s=1, \ldots, k$, are linearly independent if the lines $\mathbb{C} b_{s}$ are distinct (the Vandermonde determinant).

We conclude this section with remarks on the number of zeros in $\mathbb{S}^{2}$ of functions in $\mathcal{H}_{n}$. Let $f \in \mathcal{H}_{n}, u=\operatorname{Re} f, v=\operatorname{Im} f$. A zero of $f$ is a common zero of $u$ and $v$. The following proposition was proved in [11] in a slightly more general form. We say that $u$ is regular if zero is not a critical value for $u$.

Proposition 2 (see [1]). Suppose $n>0, u \in \mathcal{H}_{n}$. If $u$ is regular, then for any $v \in \mathcal{H}_{n}$ each connected component of $N_{u}$ contains at least two points of $N_{v}$.

The assertion follows from the Green formula, which implies that

$$
\int_{C} v \frac{\partial u}{\partial n} d s=0
$$

where $C$ is a component of $N_{u}$ ( $N_{u}$ is a Jordan contour because $u$ is regular), $d s$ is the length measure on $C$, and $\frac{\partial u}{\partial n}$ is the normal derivative; note that $\frac{\partial u}{\partial n}$ keeps its sign on $C$. For the standard sphere $\mathbb{S}^{2}$, (16) follows from the classical Green formula for the domain $D_{\varepsilon}=(1-\varepsilon, 1+\varepsilon) \times \mathbb{S}^{2}$, where $\varepsilon \in(0,1)$, and for the extensions of $u, v$ to $D_{\varepsilon}$ that are homogeneous of degree 0 .

Let $u, v \in \mathcal{H}_{n}$ be real and regular. Set

$$
\nu(u, v)=\operatorname{card} N_{u} \cap N_{v} .
$$


For singular $u, v$, the zeros must be counted with their multiplicities; if $u, v \in \mathcal{H}_{n}$, then the multiplicity of a zero can be defined as the number of smooth nodal lines that meet at it; if $u, v$ have multiplicities $k, l$ at their common zero, then we must count it $k l$ times (the greatest number of common zeros that arise under small perturbations). If $u=\phi_{a}$ with $a \in \mathbb{S}^{2}$, then $N_{u}$ is the union of $n$ parallel circles $\langle x, a\rangle=t_{k}, x \in \mathbb{S}^{2}$, where $k=1, \ldots, n$ and $t_{1}, \ldots, t_{n}$ are the zeros of $P_{n}(t)$. Since they are distinct, $P_{n}^{\prime}\left(t_{k}\right) \neq 0$ for all $k$. Proposition 2 shows that

$$
\nu\left(\phi_{a}, v\right) \geq 2 n,
$$

for any real $v \in \mathcal{H}_{n}$, where $a \in \mathbb{S}^{2}$. If $b \in \mathbb{S}^{2}$ is sufficiently close to $a$, then equality occurs for $v=\phi_{b}$. In the inequality above, $\phi_{a}$ and $n$ may be replaced with any regular $u$ and the number of components of $N_{u}$, respectively. The latter can be less than $n$ (in accordance with [12, it can be equal to one or two if $n$ is odd or even, respectively) 2 However, computer experiments support the following conjecture: $\nu(u, v) \geq 2 n$ for all real $u, v \in \mathcal{H}_{n}$.

The common zeros must be counted with their multiplicities. Otherwise, there is a simple example of two harmonics with only two common zeros: $\operatorname{Re}\left(x_{1}+i x_{2}\right)^{n}$ and $\operatorname{Im}\left(x_{1}+i x_{2}\right)^{n}$.

On the other hand, for generic real $u, v \in \mathcal{H}_{n}$ there is a trivial sharp upper bound for $\nu(u, v)$. We prove a version that is a bit stronger.

Proposition 3. Let $u, v \in \mathcal{H}_{n}$ be real. If $\nu(u, v)$ is finite, then

$$
\nu(u, v) \leq 2 n^{2} .
$$

By the Bézout theorem, if $u, v \in \mathcal{P}_{n}^{3}$ have no proper common divisor, then the set $\left\{z \in \mathbb{C}^{3}: u(z)=v(z)=0\right\}$ is the union of $n^{2}$ (with multiplicities) complex lines. Then $\nu(u, v) \leq 2 n^{2}$ because each line has at most two common points with $\mathbb{S}^{2}$. The proposition is not an immediate consequence of this fact because $u$ and $v$ may have a nontrivial common divisor with a finite number of zeros in $\mathbb{S}^{2}$. This cannot happen for $u, v \in \mathcal{H}_{n}$ by the following lemma.

Lemma 4. Suppose $u \in \mathcal{H}_{n}$ is real, $x \in \mathbb{S}^{2}$, and $u(x)=0$. Suppose $u=v w$, where $v \in \mathcal{P}_{m}^{3}$ and $w \in \mathcal{P}_{n-m}^{3}$ are real. If $w(y) \neq 0$ for all $y \in \mathbb{S}^{2} \backslash\{x\}$ sufficiently close to $x$, then $w(x) \neq 0$.

Proof. We may assume $x=(0,0,1)$. If $u$ has a zero of multiplicity $k$ at $x$, then

$$
u\left(x_{1}, x_{2}, x_{3}\right)=p_{k}\left(x_{1}, x_{2}\right) x_{3}^{n-k}+p_{k+1}\left(x_{1}, x_{2}\right) x_{3}^{n-k-1}+\cdots+p_{n}\left(x_{1}, x_{2}\right),
$$

where $p_{j} \in \mathcal{P}_{j}^{2}, p_{k} \neq 0$. Since $\Delta u=0$, we have $\Delta p_{k}=0$. Hence,

$$
p_{k}\left(x_{1}, x_{2}\right)=\operatorname{Re}\left(\lambda\left(x_{1}+i x_{2}\right)^{k}\right)
$$

for some $\lambda \in \mathbb{C} \backslash\{0\}$. Therefore, $p_{k}$ is the product of $k$ distinct linear forms. Let

$$
\begin{aligned}
w & =q_{l}\left(x_{1}, x_{2}\right) x_{3}^{n-m-l}+q_{l+1}\left(x_{1}, x_{2}\right) x_{3}^{n-m-l-1}+\cdots+q_{n-m}\left(x_{1}, x_{2}\right), \\
v & =r_{s}\left(x_{1}, x_{2}\right) x_{3}^{m-s}+r_{s+1}\left(x_{1}, x_{2}\right) x_{3}^{m-s-1}+\cdots+r_{m}\left(x_{1}, x_{2}\right),
\end{aligned}
$$

where $q_{j}, r_{j} \in \mathcal{P}_{j}^{2}$ and $q_{l}, r_{s} \neq 0$. Since $p_{k}=q_{l} r_{s}$, we have $k=l+s$; moreover, either $q_{l}$ is constant or it is a product of distinct linear forms. The latter implies that it changes its sign near $x$; then the same is true for $w$, contradictory to the assumption. Hence, $l=0$. Thus, $q_{l} \neq 0$ implies $w(x)=q_{l}(x) \neq 0$.

\footnotetext{
${ }^{2}$ The corresponding harmonic is a small perturbation of the function $\operatorname{Re}\left(x_{1}+i x_{2}\right)^{n}$.
} 
Proof of Proposition 3. Let $u, v \in \mathcal{H}_{n}$ be real, and let $w$ be the greatest common divisor of $u$ and $v$. Clearly, $w$ is real. Since $N_{u} \cap N_{v}$ is finite, the zeros of $w$ in $\mathbb{S}^{2}$ must be isolated; by Lemma $4, w$ has no zeros in $\mathbb{S}^{2}$. Applying the Bézout theorem to $u / w$ and $v / w$, we get the claim.

Equality in (17) occurs, for example, for the following pairs and their small perturbations:

$$
\begin{aligned}
& u=\phi_{a}, \quad v=\operatorname{Re}\left(x_{2}+i x_{3}\right)^{n}, \quad \text { where } \quad a=(1,0,0) ; \\
& u=\operatorname{Re}\left(i x_{2}+x_{3}\right)^{n}, \quad v=\operatorname{Re}\left(x_{1}+i x_{2}\right)^{n} .
\end{aligned}
$$

Corollary 3. If the number of critical points for real $u \in \mathcal{H}_{n}$ is finite, then it does not exceed $2 n^{2}$; in particular, this is true for a generic real $u \in \mathcal{H}_{n}$.

Proof. If $x$ is a critical point of $u$, then $\xi u(x)=0$ for any vector field $\xi \in \operatorname{so}(3)$. It is possible to choose two fields $\xi, \eta \in \mathrm{so}(3)$ that do not annihilate $u$ and are independent at all critical points; then the critical points of $u$ are precisely the common zeros of $\xi u, \eta u \in \mathcal{H}_{n}$.

Remark 4. This bound is not sharp. At least, for $n=1,2$, the number of critical points is equal to $2\left(n^{2}-n+1\right)$ provided it is finite. Let $u, v$ be as in (18). Then $u+\varepsilon v$, where $\varepsilon$ is small, has $2\left(n^{2}-n+1\right)$ critical points. I know of no example of a spherical harmonic with a greater (finite) number of critical points.

Remark 5. The consideration above proves a bit more than Corollary 3 says. A nontrivial orbit of $u$ under $\mathrm{SO}(3)$ is either 3-dimensional or 2-dimensional, and the latter occurs if and only if $u=c \phi_{a}$ for some constant $c$ and $a \in \mathbb{S}^{2}$. In the first case, the set $C$ of critical points of $u$ is precisely the set of common zeros of three linearly independent spherical harmonics (a base for the tangent space to the orbit of $u$ ). Hence, $\operatorname{codim} \mathcal{N}_{C} \geq 3$. Note that generic three harmonics have no common zero. Thus, the configuration of critical points is always degenerate. The problem of estimation of the number of critical points, components of nodal sets, nodal domains, etc. for spherical harmonics on $\mathbb{S}^{2}$ was stated in [2].

Proposition 4. The set $\mathcal{I}$ of functions $f=u+i v \in \mathcal{H}_{n}$ such that $\nu(u, v)=\infty$ is closed and nowhere dense in $\mathcal{H}_{n}$.

Proof. If the set $N_{u} \cap N_{v}$ is infinite, then it contains a Jordan arc that extends to a contour, because $u$ and $v$ are real analytic (by [6], a nodal set, outside of its finite subset, is a finite union of smooth arcs). This contour cannot be included into a disk $D$ contained in some of the nodal domains: otherwise, the corresponding first Dirichlet eigenvalue would be greater than $n(n+1)$. Therefore, the diameter of the contour is bounded from below. This implies that the set $\mathcal{I}$ is closed. If $f \in \mathcal{I}$, then $u$ and $v$ have a nontrivial common divisor by the Bézout theorem; hence, $\mathcal{I}$ is nowhere dense.

In all examples known to me, if $f \in \mathcal{I}$, then $N_{u} \cap N_{v}$ is a union of circles.

\section{§3. Estimates OF NODAL LENGTHS AND INNER RADII}

Let $M$ be a $C^{\infty}$-smooth compact connected Riemannian manifold, let $m=\operatorname{dim} M$, and let $\mathfrak{h}^{k}$ be the $k$-dimensional Hausdorff measure on $M$. Yau conjectured that there exist positive constants $c$ and $C$ such that

$$
c \sqrt{\lambda} \leq \mathfrak{h}^{m-1}\left(N_{u}\right) \leq C \sqrt{\lambda}
$$

for the nodal set $N_{u}$ of any eigenfunction $u$ corresponding to the eigenvalue $-\lambda$. For real analytic $M$, this conjecture was proved by Donnelly and Fefferman in 8 . In the 
case of a surface, lower bounds were obtained in [5] and [18; in [18] it was shown that $c=\frac{1}{11} \operatorname{Area}(M)$ fits.

First, we consider the case where $M=\mathbb{S}^{m} \subset \mathbb{R}^{m+1}, m \geq 1$. Set

$$
\psi(x)=\operatorname{Re}\left(x_{1}+i x_{2}\right)^{n} .
$$

Clearly, $\psi \in \mathcal{H}_{n}^{m+1}$. Let $\phi$ denote a zonal spherical harmonic; we omit the index because the geometric quantities that characterize $N_{\phi}$ are independent of it. Set

$$
\omega_{k}=\mathfrak{h}^{k}\left(\mathbb{S}^{k}\right)=\frac{2 \pi^{\frac{k+1}{2}}}{\Gamma\left(\frac{k+1}{2}\right)} .
$$

Theorem 3. For any nonzero real $u \in \mathcal{H}_{n}^{m+1}$, we have

$$
\mathfrak{h}^{m-1}\left(N_{u}\right) \leq \mathfrak{h}^{m-1}\left(N_{\psi}\right)=n \omega_{m-1} .
$$

The theorem is simply an observation modulo the following fact (a particular case of Theorem 3.2.48 in [10]). A set that can be realized as the image of a bounded subset of $\mathbb{R}^{k}$ under a Lipschitz mapping is said to be $k$-rectifiable (we only consider sets that can be realized as a countable union of compact sets). Since $u \in \mathcal{H}_{n}^{m+1}$ is a polynomial, the set $N_{u}$ is $(m-1)$-rectifiable. Let $\mu_{m}$ denote the invariant measure on $\mathrm{O}(m+1)$ with total mass 1 .

Theorem 4 (see [10]). Suppose $A, B \subseteq \mathbb{S}^{d}$ are compact, $A$ is $k$-rectifiable, and $B$ is $l$-rectifiable. Set $r=k+l-d$. Suppose $r \geq 0$. Then

$$
\int_{\mathrm{O}(d)} \mathfrak{h}^{r}(A \cap g B) d \mu_{d}(g)=K \mathfrak{h}^{k}(A) \mathfrak{h}^{l}(B),
$$

where $K=\frac{\Gamma\left(\frac{k+1}{2}\right) \Gamma\left(\frac{l+1}{2}\right)}{2 \Gamma\left(\frac{1}{2}\right)^{d} \Gamma\left(\frac{r+1}{2}\right)}=\frac{\omega_{r}}{\omega_{k} \omega_{l}}$.

If $r=0$, then the left-hand side of (20) is a version of the Favard measure for spheres (on $A$ or $B$ ). Also, note that in this setting (20) can be proved directly, because, for fixed $A$ (or $B$ ), the left-hand side is additive on finite families of disjoint compact sets; thus, it suffices to check its asymptotic behavior on small pieces of submanifolds.

Lemma 5. For any real $u \in \mathcal{H}_{n}^{m+1}$ and each big circle $\mathbb{S}^{1}$ in $\mathbb{S}^{m}$, if $\mathbb{S}^{1} \cap N_{u}$ is finite, then

$$
\operatorname{card}\left(\mathbb{S}^{1} \cap N_{u}\right) \leq 2 n .
$$

Proof. The restriction of $u$ to the linear span of $\mathbb{S}^{1}$, which is 2-dimensional, is a homogeneous polynomial of degree $n$ in two variables.

Proof of Theorem 3. Since $\mathbb{S}^{1}$ has precisely two points in common with any hyperplane that does not contain it, for almost all $g \in \mathrm{O}(m+1)$ we have

$$
\operatorname{card}\left(g \mathbb{S}^{1} \cap N_{u}\right) \leq 2 n=\operatorname{card}\left(g \mathbb{S}^{1} \cap N_{\psi}\right) .
$$

Integrating over $\mathrm{O}(m+1)$ and applying (20) with $k=1, l=m-1, A=\mathbb{S}^{1}, B=N_{u}$, and $B=N_{\psi}$, we get the inequality in (19). The identity in (19) is evident.

A lower bound can also be obtained in a similar way. In what follows, we assume that $k=l=1$ and $m=2$; then $K=\frac{1}{2 \pi^{2}}$, and (19) reads as follows:

$$
\mathfrak{h}^{1}\left(N_{u}\right) \leq 2 \pi n .
$$

The nodal set $N_{\phi}$ of a zonal spherical harmonic $\phi=\phi_{a} \in \mathcal{H}_{n}$, where $a \in \mathbb{S}^{2}$, is the union of parallel circles of Euclidean radii $\sqrt{1-t_{k}^{2}}$, where the $t_{k}$ are the zeros of the 
Legendre polynomial $P_{n}$. The smallest circle corresponds to the greatest zero $t_{n}$. Set $r_{n}=\sqrt{1-t_{n}^{2}}$ and let $C_{n}$ be a circle in $\mathbb{S}^{2}$ of Euclidean radius $r_{n}$. By Proposition 2, for any $u \in \mathcal{H}_{n}$, we have

$$
\operatorname{card}\left(g C_{n} \cap N_{u}\right) \geq 2 \text { for all } g \in \mathrm{O}(3) .
$$

By (20),

$$
\mathfrak{h}^{1}\left(N_{u}\right) \geq \frac{2 \pi}{r_{n}} .
$$

By [21, Theorem 6.3.4], $t_{n}=\cos \theta_{n}$ with

$$
0<\theta_{n}<\frac{j_{0}}{n+\frac{1}{2}}
$$

and $j_{0} \approx 2.4048$ is the smallest positive zero of the Bessel function $J_{0}$. Relation 21, (6.3.15)] shows that this estimate is asymptotically sharp: $\lim _{n \rightarrow \infty} n \theta_{n}=j_{0}$. Thus,

$$
r_{n}=\sin \theta_{n}<\sin \frac{j_{0}}{n+\frac{1}{2}}<\frac{j_{0}}{n+\frac{1}{2}}
$$

and we get

$$
\mathfrak{h}^{1}\left(N_{u}\right)>\frac{2 \pi}{j_{0}}\left(n+\frac{1}{2}\right) .
$$

The bound (25) is not the best possible, but it is greater than $\frac{1}{11}$ Area $(M) \sqrt{\lambda}$, the bound of the paper [18]:

$$
\frac{4 \pi}{11} \sqrt{n(n+1)}<\frac{2 \pi}{j_{0}}\left(n+\frac{1}{2}\right)
$$

because $\frac{4 \pi}{11} \approx 1.4248, \frac{2 \pi}{j_{0}} \approx 2.6127$; in accordance with [18, $\frac{1}{11}$ Area $(M) \sqrt{\lambda}$ estimates from below the nodal length for all closed Riemannian surfaces $M$ (for sufficiently large $\lambda$ in general, and for all $\lambda$ if the curvature is nonnegative). It seems plausible that the sharp lower bound may coincide with the length of the nodal set of a zonal harmonic. By [21, (6.21.5)], we have $\frac{k-\frac{1}{2}}{n+\frac{1}{2}} \pi \leq \tau_{n-k} \leq \frac{k}{n+\frac{1}{2}} \pi$, where $\cos \tau_{k}, k=0, \ldots, n-1$, are the zeros of $P_{n}$ in decreasing order (i.e., $\tau_{1}=\theta_{n}$ ). Hence,

$$
\mathfrak{h}^{1}\left(N_{\phi}\right)=2 \pi \sum_{k=1}^{n} \sin \theta_{k} \approx 2 \pi n \int_{0}^{1} \sin \pi x d x=4 n
$$

as $n \rightarrow \infty$. If the conjecture above is true, then the upper bound is rather close to the lower one, because their ratio tends to $\frac{\pi}{2}$ as $n \rightarrow \infty$.

It is also possible to estimate the inner radius of $\mathbb{S}^{2} \backslash N_{u}$ :

$$
\operatorname{inr}\left(\mathbb{S}^{2} \backslash N_{u}\right)=\sup \left\{\inf _{y \in N_{u}} \rho(x, y): x \in \mathbb{S}^{2}\right\},
$$

where $\rho$ is the inner metric in $\mathbb{S}^{2}$ :

$$
\rho(x, y)=\arccos \langle x, y\rangle .
$$

The least upper bound is evident:

$$
\operatorname{inr}\left(\mathbb{S}^{2} \backslash N_{u}\right) \leq \operatorname{inr}\left(\mathbb{S}^{2} \backslash N_{\phi}\right)=\theta_{n},
$$

by (24). Indeed, equality is attained for $u=\phi$, and the left-hand side cannot be greater because the circle $C_{n}$ intersects any nodal set by Proposition 2. Let $C(\theta)$ be a circle of radius $\theta$ in the inner metric of $\mathbb{S}^{2}$; then the Euclidean radius of $C(\theta)$ is $r=\sin \theta$. A number $\theta_{0}>0$ is a lower bound for the inner radius if and only if the following conditions are fulfilled:

(i) $\theta_{0} \leq \theta_{n}$; 
(ii) for each real $u \in \mathcal{H}_{n}$, there exists $g \in \mathrm{O}(3)$ such that $g C\left(\theta_{0}\right) \cap N_{u}=\varnothing$ (note that the disk bounded by $C\left(\theta_{0}\right)$ cannot contain a component of $N_{u}$, by (i)). Next, for almost all $g \in \mathrm{O}(3)$ the number $\operatorname{card}\left(g C\left(\theta_{0}\right) \cap N_{u}\right)$ is even. Therefore, we may assume that

$$
\operatorname{card}\left(g C\left(\theta_{0}\right) \cap N_{u}\right) \geq 2
$$

if $g C\left(\theta_{0}\right) \cap N_{u} \neq \varnothing$. Set $r_{0}=\sin \theta_{0}$. If (ii) is false, then

$$
2 \leq \frac{1}{2 \pi^{2}} \mathfrak{h}^{1}\left(C\left(\theta_{0}\right)\right) \mathfrak{h}^{1}\left(N_{u}\right)=\frac{r_{0}}{\pi} \mathfrak{h}^{1}\left(N_{u}\right) \leq 2 r_{0} n
$$

by (20). Thus, if $r_{0}<\frac{1}{n}$, then $\theta_{0}$ is a lower bound for $\operatorname{inr}\left(\mathbb{S}^{2} \backslash N_{u}\right)$. Hence $\arcsin \frac{1}{n}$ is a lower bound for $\operatorname{inr}\left(\mathbb{S}^{2} \backslash N_{u}\right)$. This estimate seems to be not sharp; perhaps, the set $\mathbb{S}^{2} \backslash N_{\psi}$ has the smallest inner radius (it is equal to $\frac{\pi}{2 n}$ ).

We summarize the results on $\mathbb{S}^{2}$.

Theorem 5. Let $M=\mathbb{S}^{2}$. For any nonzero real $u \in \mathcal{H}_{n}$, we have

$$
\begin{aligned}
& \frac{2 \pi}{j_{0}}\left(n+\frac{1}{2}\right)<\mathfrak{h}^{1}\left(N_{u}\right) \leq 2 \pi n, \\
& \arcsin \frac{1}{n} \leq \operatorname{inr}\left(\mathbb{S}^{2} \backslash N_{u}\right) \leq \theta_{n}<\frac{j_{0}}{n+\frac{1}{2}} .
\end{aligned}
$$

In (26), the upper bound is attained if $u=\psi$; the upper bound $\theta_{n}$ in (27) is attained for $u=\phi$.

\section{§4. Mean Hausdorff measure of intersections of nodal SETS}

We fix $m \geq 2$ and the unit sphere $\mathbb{S}^{m} \subset \mathbb{R}^{m+1}$. We shall find the mean value over $u_{1}, \ldots, u_{k}, k \leq m$, of the Hausdorff measure of the sets

$$
N_{u_{1}} \cap \cdots \cap N_{u_{k}} \subset \mathbb{S}^{m} \text {. }
$$

If $k=m$, then this is the mean number of the common zeros of $u_{1}, \ldots, u_{m}$ in $\mathbb{S}^{m}$. Set

$$
\mathbf{n}=\left(n_{1}, \ldots, n_{k}\right), \quad \delta(n)=\operatorname{dim} \mathcal{H}_{n}^{m+1}-1,
$$

where $n, n_{j}$ are positive integers. We define the mean value as follows:

$$
M_{\mathbf{n}}=\int_{\mathbb{S}^{\delta\left(n_{1}\right) \times \cdots \times \mathbb{S}^{\delta\left(n_{k}\right)}}} \mathfrak{h}^{m-k}\left(N_{u_{1}} \cap \cdots \cap N_{u_{k}}\right) d \tilde{\sigma}_{\delta\left(n_{1}\right)}\left(u_{1}\right) \cdots d \tilde{\sigma}_{\delta\left(n_{k}\right)}\left(u_{k}\right),
$$

where $\tilde{\sigma}_{j}$ denotes the invariant measure on $\mathbb{S}^{j}$ with total mass 1 . Let $\lambda_{n}$ be the eigenvalue of $-\Delta$ in $\mathcal{H}_{n}^{m+1}$; we recall that

$$
\lambda_{n}=n(n+m-1) .
$$

Theorem 6. Let $1 \leq k \leq m$. Then

$$
M_{\mathbf{n}}=\omega_{m-k} m^{-\frac{k}{2}} \sqrt{\lambda_{n_{1}} \cdots \lambda_{n_{k}}}
$$

where $M_{\mathbf{n}}$ is defined by (28).

If $k=m$, then we get the mean value of card $\left(N_{u_{1}} \cap \cdots \cap N_{u_{m}}\right)$; since $\omega_{0}=2$ and $\mathfrak{h}^{0}=$ card, it is equal to $2 m^{-\frac{m}{2}} \sqrt{\lambda_{n_{1}} \cdots \lambda_{n_{m}}}$.

There is a natural equivariant immersion $\iota_{n}: \mathbb{S}^{m} \rightarrow \mathbb{S}^{\delta(n)} \subset \mathcal{H}_{n}^{m+1}$ :

$$
\iota_{n}(a)=\frac{\phi_{a}}{\left|\phi_{a}\right|}
$$

If $n$ is odd, then $\iota_{n}$ is one-to-one; for even $n>0, \iota_{n}$ is a two-sheeted covering that identifies opposite points. Clearly, the Riemannian metric in $\iota\left(\mathbb{S}^{m}\right)$ is $\mathrm{O}(m+1)$-invariant, and the 
stable subgroup of $a$ acts transitively on the spheres in $T_{a} \mathbb{S}^{m}$. Hence, the mapping $\iota_{n}$ is locally a metric homothety. Let $s_{n}$ be its coefficient. Clearly,

$$
s_{n}=\frac{\left|d_{a} \iota_{n}(v)\right|}{|v|},
$$

where the right-hand side is independent of $a \in \mathbb{S}^{m}$ and $v \in T_{a} \mathbb{S}^{m} \backslash\{0\}$. For any $l$-rectifiable set $X \subseteq \mathbb{S}^{m}$ such that $X \cap(-X)=\varnothing$, where $l \leq m$, we have

$$
\mathfrak{h}^{l}\left(\iota_{n}(X)\right)=s_{n}^{l} \mathfrak{h}^{l}(X)
$$

Lemma 6. Let $u \in \mathcal{H}_{n}^{m+1}$, and let $X \subseteq \mathbb{S}^{m}$ be compact, symmetric, and $(r+1)$-rectifiable, where $r \leq m-1$. Then

$$
\int_{\mathbb{S}^{\delta(n)}} \mathfrak{h}^{r}\left(N_{u} \cap X\right) d \sigma_{\delta(n)}(u)=s_{n} \frac{\omega_{r}}{\omega_{r+1}} \mathfrak{h}^{r+1}(X) .
$$

Proof. Since both sides are additive with respect to $X$, we may assume $X \cap(-X)=\varnothing$. We apply Theorem 4 to the sphere $\mathbb{S}^{\delta(n)}$ and its subsets $A=\mathbb{S}^{\delta(n)-1}$ and $B=\iota_{n}(X)$. In the notation of that theorem, $d=\delta(n), k=d-1, l=r+1 ; K \omega_{k}=\frac{\omega_{r}}{\omega_{l}}$. Replacing integration over $\mathbb{S}^{d}$ by averaging over $\mathrm{O}(d+1)$ and using (32) , we get

$$
\begin{aligned}
\int_{\mathbb{S}^{d}} \mathfrak{h}^{r} & \left(N_{u} \cap X\right) d \sigma_{d}(u)=\frac{1}{s_{n}^{r}} \int_{\mathbb{S}^{d}} \mathfrak{h}^{r}\left(\iota\left(N_{u} \cap X\right)\right) d \sigma_{d}(u) \\
= & \frac{1}{s_{n}^{r}} \int_{\mathbb{S}^{d}} \mathfrak{h}^{r}\left(u^{\perp} \cap \iota(X)\right) d \sigma_{d}(u)=\frac{1}{s_{n}^{r}} \int_{\mathrm{O}(d+1)} \mathfrak{h}^{r}\left(g \mathbb{S}^{k} \cap \iota(X)\right) d \mu_{d}(g) \\
= & \frac{1}{s_{n}^{r}} K \mathfrak{h}^{k}\left(\mathbb{S}^{k}\right) \mathfrak{h}^{r+1}(\iota(X))=\frac{\omega_{r}}{s_{n}^{r} \omega_{r+1}} \mathfrak{h}^{r+1}(\iota(X))=s_{n} \frac{\omega_{r}}{\omega_{r+1}} \mathfrak{h}^{r+1}(X) .
\end{aligned}
$$

Corollary 4. The mean value of $\mathfrak{h}^{m-1}\left(N_{u}\right)$ over $u \in \mathcal{H}_{n}^{m+1}$ is equal to $s_{n} \omega_{m-1}$.

Proof. Set $X=\mathbb{S}^{m}, r=m-1$.

Corollary 5. Let $M_{\mathbf{n}}, m$, and $k$ be as in (28). Then

$$
M_{\mathbf{n}}=\omega_{m-k} \prod_{j=1}^{k} s_{n_{j}} .
$$

Proof. Set $X=N_{u_{1}} \cap \cdots \cap N_{u_{k-1}}$. By Lemma 6 ,

$$
M_{\mathbf{n}}=s_{n_{k}} \frac{\omega_{m-k}}{\omega_{m-k+1}} M_{\mathbf{n}^{\prime}},
$$

where $\mathbf{n}^{\prime}=\left(n_{1}, \ldots, n_{k-1}\right)$. Applying this procedure repeatedly and using Corollary 4 at the final step, we get (33).

It remains to find $s_{n}$. Set $d=\operatorname{dim} \mathrm{O}(m+1)$. Since the stable subgroup $\mathrm{O}(m)$ of the point $a=(0, \ldots, 0,1)$ acts transitively on the spheres in $T_{a} \mathbb{S}^{m}$, the invariant Riemannian metric in $\mathbb{S}^{m}$ can be lifted up to a bi-invariant metric on $\mathrm{O}(m+1)$ in such a way that the canonical projection $\mathrm{O}(m+1) \rightarrow \mathbb{S}^{m}$ is a metric submersion. Let $\xi_{1}, \ldots, \xi_{m}, \ldots, \xi_{d}$ be an orthonormal linear basis in the Lie algebra so $(m+1)$. Realizing $\operatorname{so}(m+1)$ by the left invariant vector fields on $\mathrm{O}(m+1)$, we get the invariant Laplace-Beltrami operator on $\mathrm{O}(m+1)$ :

$$
\tilde{\Delta}=\xi_{1}^{2}+\cdots+\xi_{d}^{2}
$$

The sum is independent of the choice of the basis because it is left invariant and this property holds true at the identity element $e$. Thus, we may assume that

$$
\xi_{m+1}, \ldots, \xi_{d} \in \mathrm{so}(m)
$$


For $f \in C^{2}\left(\mathbb{S}^{m}\right)$, we set $\tilde{f}(g)=f(g a)$. Then $\left\langle\Delta f, \phi_{a}\right\rangle=\tilde{\Delta} \tilde{f}(e)$. Since $\iota$ is equivariant, we have

$$
d_{a} \iota(\xi a)=\frac{1}{\left|\phi_{a}\right|} \xi \phi_{a}
$$

for all $\xi \in \operatorname{so}(m+1)$. From (34) it follows that $\xi_{1} a, \ldots, \xi_{m} a$ is a basis for $T_{a} \mathbb{S}^{m}$, and $\xi_{1} \phi_{a}, \ldots, \xi_{m} \phi_{a}$ is a basis for $T_{\phi_{a}} \iota\left(\mathbb{S}^{m}\right)$. Moreover,

$$
\begin{aligned}
\left|\xi_{k} a\right| & =1, \quad k=1, \ldots, m, \\
\xi_{k} a & =0, \quad k=m+1, \ldots, d,
\end{aligned}
$$

where the first relation is true because the projection $\mathrm{O}(m+1) \rightarrow \mathbb{S}^{m}$ is a metric submersion. Using these identities and also (30), (31), and (35), we get

$$
\begin{aligned}
m s_{n}^{2} & =s_{n}^{2} \sum_{k=1}^{d}\left|\xi_{k} a\right|^{2}=\sum_{k=1}^{d}\left|d_{a} \iota\left(\xi_{k} a\right)\right|^{2}=\frac{1}{\left|\phi_{a}\right|^{2}} \sum_{k=1}^{d}\left|\xi_{k} \phi_{a}\right|^{2} \\
& =-\frac{1}{\left|\phi_{a}\right|^{2}} \sum_{k=1}^{d}\left\langle\xi_{k}^{2} \phi_{a}, \phi_{a}\right\rangle=-\frac{1}{\left|\phi_{a}\right|^{2}}\left\langle\Delta \phi_{a}, \phi_{a}\right\rangle=\lambda_{n} .
\end{aligned}
$$

Proof of Theorem 6. In accordance with the calculation above,

$$
s_{n}=\sqrt{\frac{\lambda_{n}}{m}} .
$$

Thus, Corollary [5] implies (29).

In the case where $n_{1}=\cdots=n_{k}=n$, there is another natural explanation of formulas (29) and (33):

$$
M_{\mathbf{n}}=\omega_{m-k}\left(\frac{\lambda_{n}}{m}\right)^{\frac{k}{2}}=\omega_{m-k} s_{n}^{k} .
$$

The mean value can be defined as the average over the action of the group $\mathrm{O}(m+1)$ on the set of subspaces of codimension $k$ in $\mathcal{H}_{n}^{m+1}$, which can be realized as $\mathcal{N}_{u_{1}} \cap \cdots \cap \mathcal{N}_{u_{k}}=$ $u_{1}^{\perp} \cap \cdots \cap u_{k}^{\perp}$ :

$$
\begin{aligned}
M_{\mathbf{n}} & =\int_{\mathrm{O}(m+1)} \mathfrak{h}^{m-k}\left(\iota_{n}^{-1}\left(g \mathbb{S}^{\delta(n)-k} \cap \iota_{n}\left(\mathbb{S}^{m}\right)\right)\right) d \mu_{m}(g) \\
& =s_{n}^{k-m} \int_{\mathrm{O}(m+1)} \mathfrak{h}^{m-k}\left(g \mathbb{S}^{\delta(n)-k} \cap \iota_{n}\left(\mathbb{S}^{m}\right)\right) d \mu_{m}(g) \\
& =s_{n}^{k-m} \frac{\omega_{m-k}}{\omega_{m}} \mathfrak{h}^{m}\left(\iota\left(\mathbb{S}^{m}\right)\right)=\omega_{m-k} s_{n}^{k} .
\end{aligned}
$$

The method of calculation of the mean Hausdorff measure can easily be extended to families of invariant (possibly, reducible) finite-dimensional function spaces on a homogeneous space whose isotropy group acts transitively on the spheres in the tangent space.

\section{ACKNOWLEDGMENTS}

I am grateful to D. Jakobson for useful references and comments and to L. Polterovich for his question/conjecture on "the Bézout theorem in the mean". 


\section{REFERENCES}

[1] V. I. Arnol'd, Lectures on partial differential equations, "FAZIS", Moscow, 1997; English transl., Springer-Verlag, Berlin, 2004. MR.1641652 (2000e:35001) MR2031206 (2004j:35002)

[2] V. I. Arnol'd et al., Some unsolved problems in the theory of differential equations and mathematical physics, Uspekhi Mat. Nauk 44 (1989), no. 4, 191-202; English transl., Russian Math. Surveys 44 (1989), no. 4, 157-171. MR1023106 (90m:00003)

[3] A. Erdélyi, W. Magnus, F. Oberhettinger, and F. G. Tricomi, Higher transcendental functions. Vol. II, McGraw-Hill Book Co., Inc., New York, 1953. MR0058756 (15:419i)

[4] P. Bérard, Volume des ensembles nodaux des fonctions propres du laplacien, Bony-SjöstrandMeyer Seminar, 1984-1985, École Polytech., Palaiseau, 1985, Exp. No. 14. MR 0819780|(87e:58202)

[5] J. Brüning, Über Knoten von Eigenfunktionen des Laplace-Beltrami Operators, Math. Z. 158 (1978), 15-21. MR0478247 (57:17732)

[6] S.-Y. Cheng, Eigenfunctions and nodal sets, Comment. Math. Helv. 51 (1976), 43-55. MR0397805 $(53: 1661)$

[7] R. Courant and D. Hilbert, Methoden der mathematischen Physik. Bd. 1, Grundlehren Math. Wiss., Bd. 12, Springer-Verlag, Berlin, 1931.

[8] H. Donnelly and C. Fefferman, Nodal sets of eigenfunctions on Riemannian manifolds, Invent. Math. 93 (1988), no. 1, 161-183. MR0943927 (89m:58207)

[9] A. Eremenko, D. Jakobson, and N. Nadirashvili, On nodal sets and nodal domains on $\mathbb{S}^{2}$ and $\mathbb{R}^{2}$, Preprint arXiv:math.SP/0611627.

[10] H. Federer, Geometric measure theory, Grundlehren Math. Wiss., Bd. 153, Springer, Berlin, 1969. MR0257325 (41:1976)

[11] V. M. Gichev, A note on common zeros of Laplace-Beltrami eigenfunctions, Ann. Global Anal. Geom. 26 (2004), 201-208. MR2071488 (2005d:58053)

[12] H. Lewy, On the minimum number of domains in which the nodal lines of spherical harmonics divide the sphere, Comm. Partial Differential Equations 2 (1977), no. 12, 1233-1244. MR.0477199 $(57: 16740)$

[13] D. Mangoubi, On the inner radius of a nodal domain, arXiv:math/0511329v3. Canad. Math. Bull. 5 (2008), 249-260. MR2414212

[14] Local asymmetry and the inner radius of nodal domains, arXiv:math/0703663v3. Comm. Partial Differential Equations 33 (2008), 1611-1621. MR2450173

[15] J. C. Maxwell, A treatise on electricity and magnetism. Vol. 1, Dover Publ., Inc., New York, 1954. MR0063293 (16:99b)

[16] J. Neuheisel, The asymptotic distribution of nodal sets on spheres, Johns Hopkins Ph.D. Thesis, 2000.

[17] Z. Rudnick and I. Wigman, On the volume of nodal sets for eigenfunctions of the Laplacian on the torus, Preprint arXiv:math-ph/0609072v2. Ann. Henri Poincaré 9 (2008), 109-130. MR2389892 (2009b:58072)

[18] A. Savo, Lower bounds for the nodal length of eigenfunctions of the Laplacian, Ann. Global Anal. Geom. 19 (2001), 133-151. MR1826398 (2002g:58055)

[19] E. Stein and G. Weiss, Introduction to Fourier analysis on Euclidean spaces, Princeton Math. Ser., vol. 32, Princeton Univ. Press, Princeton, NJ, 1971. MR0304972 (46:4102)

[20] J. J. Sylvester, Note on spherical harmonics, Philos. Mag. 2 (1876), 291-307.

[21] G. Szegö, Orthogonal polynomials, Amer. Math. Soc. Colloq. Publ., vol. 23, Amer. Math. Soc., Providence, RI, 1959. MR0106295 (21:5029)

[22] S. T. Yau (ed.), Seminar on Differential Geometry, Ann. of Math. Stud., vol. 102, Princeton Univ. Press, Princeton, NJ, 1982. MR0645728 (83a:53002)

Omsk Division, Sobolev Mathematical Institute, Siberian Branch, Russian Academy of Sciences, Ul. Pevtsova 13, 644099 Omsk, Russia

E-mail address: gichev@ofim.oscsbras.ru

Received 11/SEP/2007

Translated by THE AUTHOR 